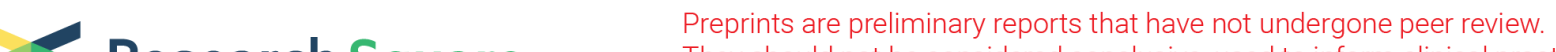 Research Square They should not be considered conclusive, used to inform clinical practice, or referenced by the media as validated information.
}

\section{Reduced Serum Cholinesterase Activity \\ Distinguishes Hepatic Encephalopathy From 48 Types of Human Diseases}

\section{Yanfei Rong ( $\square$ 1135257802@qq.com )}

Systems Biology \& Medicine Center for Complex Diseases, Center for Clinical Research, Affiliated Hospital of Qingdao University, Qingdao, 266003, China;

\section{Meng Zhang}

systems Biology खMedicine Center for Complex Diseases

\section{Yiyang Zhang}

Kuang Yaming Hornors School,Nanjing University

\section{Yongzheng Pang}

The Affiliated Hospital of Qingdao University

\section{Lijuan Zhang}

Systems Biology $\llbracket$ Medicine Center for Complex Diseases

\section{Yachong Guo}

Nanjing University

\section{Haitao Pei}

The Affiliated Hospital of Qingdao University

\section{Research}

Keywords: Serum cholinesterase activity (CHE), Hepatic encephalopathy (HE), Area under curve (AUC), Receiving operator curve (ROC), Accuracy, Sensitivity, and Specificity

Posted Date: December 15th, 2020

DOl: https://doi.org/10.21203/rs.3.rs-126761/v1

License: (c) (1) This work is licensed under a Creative Commons Attribution 4.0 International License. Read Full License 


\section{Abstract}

Background: Hepatic encephalopathy is a complication of central nervous systems due to liver failurerelated brain inflammation. Less than half of patients suffering from liver failure develop hepatic encephalopathy, which suggests other factors beyond liver failure might contribute to hepatic encephalopathy. Indeed, we reported previously that the levels of serum direct bilirubin, a liver cell-made product, are counter-intuitively highest in hepatic encephalopathy patients among 72 clinically defined diseases. In current study, we tested if cholinesterase could serve as a biomarker for hepatic encephalopathy by comparing serum cholinesterase activities among 48 different types of human diseases.

Methods: The activity of serum cholinesterase was determined by the standard "continuous monitoring method" in the clinical laboratory of the hospital where the serum cholinesterase activities from 137,305 independent tests with 48 clinically defined diseases and 3,387 independent tests from healthy individuals who came to the hospital for physical examination during the past 5 years were retrieved. All data were analyzed with RStudio V.1.3.1073 and python libraries 3.8.

Results: We found that all 48 types of diseases had decreased cholinesterase activity compared to control based on either mean or median values. Remarkably, hepatic encephalopathy had the lowest cholinesterase activity and the serum cholinesterase activity was the best biomarker for hepatic encephalopathy (AUC 0.99, sensitivity 100\%, and specificity 99\%) among all diseases. Moreover, two component analysis of cholinesterase activity distributions revealed hepatic encephalopathy resembled preeclampsia and uremia whereas cirrhosis resembled multiple myeloma, leukemia, myeloproliferative disorder, and liver cancer.

Conclusions: Decreased cholinesterase activity was an almost perfect serum biomarker for patients suffering hepatic encephalopathy at all stages. The resemblance of hepatic encephalopathy to preeclampsia and uremia based on cholinesterase activities provided new insight in understanding hepatic encephalopathy etiology beyond liver failure.

\section{Background}

Hepatic encephalopathy (HE) is known as a spectrum of neuropsychiatric abnormalities in patients suffering from liver dysfunction when other known brain disease has been excluded[1, 2]. HE is not only associated with systemic inflammation, but also with neuroinflammation [3-5] or endotoxemia[6, 7]. Its onset may be gradual or sudden manifested as neuropsychiatric disorders and motor dysfunctions[8]. The one-year mortality of severe HE is $54 \%[9]$. HE is divided into covert type including minimal HE and grade I HE, and overt type ranging from grade II to grade IV HE[10]. Increased serum ammonia[11], $\gamma^{-}$ aminobutyric acid[12], manganese[13], or oxidative/nitrosative stress, etc. are correlated to the occurrence and development of HE[14]. Lactone, rifaximin, probiotics or molecular adsorbent recirculating system that have anti-inflammatory effects can significantly improve the neuropsychiatric symptoms of patients. 
Even though $\mathrm{HE}$ is associated with liver failure, less than half of patients suffering liver failure develop $\mathrm{HE}$, suggesting other factors contributed to HE development. Indeed, we found that the levels of serum direct bilirubin, a liver cell-made product, are counter-intuitively highest in HE patients among 72 clinically defined diseases[15]. In order to monitor therapeutic effects for patients suffering from HE, blood-based biomarkers are desirable. High levels of blood ammonia are usually used to monitor the therapeutic effects of HE. However, recent studies have shown that concentrations of blood ammonia cannot always reflect the severity of $\mathrm{HE}$ [16-18]. Therefore, it is necessary to explore other serum biomarkers with better sensitivity and specificity for monitoring the therapeutic effects of HE and revealing more etiology of $\mathrm{HE}$.

Serum CHE is a serine hydrolase mainly synthesized in liver, which is also expressed in pancreas, glia, and the endothelial cells of the central nervous system. CHE consists of four identical subunits with 574 amino acids each and each monomer contains ten potential $\mathrm{N}$-glycosylation sequences in which nine are glycosylated[19, 20]. CHE is centered on a proline-rich polypeptide and the four identical subunits interweave at the $\mathrm{C}$-terminal to form the mature tetramer[21]. It comes to realize that $\mathrm{CHE}$ plays a vital role in the hydrolysis of different esters, including butyrylcholine, succinylcholine and ghrelin[22], and is also involved in the metabolism of certain drugs and toxic substance, such as procaine, cocaine, heroin[23, $24]$, aspirin, and organophosphorus poisons $[25,26]$. In addition, $\mathrm{CHE}$ is associated with inflammation and parasympathetic dysfunction[27, 28]. However, the serum $\mathrm{CHE}$ activities and their dynamic ranges have never been studied and compared systematically in different types of diseases.

In current study, the serum CHE levels from 137,305 patients with 48 clinically defined diseases and 3,387 healthy individuals during the same period were retrieved from the clinical laboratory of the Affiliated Hospital of Qingdao University during the past 5 years and different statistical methods were employed to perform data analysis.

\section{Methods}

\section{Study participants}

After obtaining approval from the Hospital Ethics Review Board of Qingdao University, we were allowed to retrieve the electronic medical records and lab data of serum $\mathrm{CHE}$ activities of both healthy individuals and patients with clinically defined diseases from the clinical laboratory of the Affiliated Hospital of Qingdao University during the past 5 years. All research was performed in accordance with relevant guidelines/regulations and informed consent was obtained from all participants and/or their legal guardians.

\section{Clinical Data Collection}

All diseases with more than 84 independent serum CHE activity tests over past 5 years were chosen. All patients with primary diagnosis of specific disease were included in current study without exclusions. Thus, all diseases include all patients at different stages of disease development with or without medical 
interventions. However, we excluded the $2.5 \%$ highest and the $2.5 \%$ lowest $\mathrm{CHE}$ activities for each disease. As results, 137,305 CHE activities from patients suffering from 48 types of diseases and 3,387 CHE activities from healthy controls were used for data analysis.

\section{Measurements Of Serum Che Activities}

Various methods have been used to determine the serum CHE activity during the past[29], but the use is limited due to their limitations. Classical spectrophotometric Ellman's method is used widely to determine activity of CHE due to its advantages of being fast, simple, cheap and accurate. The method involves two steps. Firstly, butylthiocholine is converted into butyric acid and thiocholine under the action of serum CHE. Thiocholine is then reacted with 5,5'-dithiobis-(2-nitrobenzoic) spontaneously, which produces 5thio-2-nitrobenzoic acid and 2-nitrobenzoic acid-5-thiocholine[30, 31]. The activity of CHE in the sample is calculated by measuring the increased absorbance of 5-thio-2-nitrobenzoic acid at $410 \mathrm{~nm}$. The serum CHE assay used by the clinical lab in our hospital is "the continuous monitoring method" with the same principle of the spectrometric Ellman's method.

ROC analysis. ROC curves were plotted using SPSS v26 (IBM, Armonk, US). Youden's indices were calculated using ROC curve coordinates in order to determine AUCs, accuracy, sensitivity and specificity at the point where test performance is optimal.

\section{Statistical analysis}

All data retrieved was analyzed with RStudio V.1.3.1073 (RStudio, Boston, USA) and python libraries 3.8 (Anaconda Software Distribution). The results were demonstrated as median values and means \pm standard deviation (SD). Standard t-test was used to compare the clinical characteristics of subjects in the specific disease and control groups. Median levels of serum $\mathrm{CHE}$ activities between groups were compared by means of the Mann-Whitney U-test. Groups were compared using the Kruskal-Wallis test (a non-parametric one-way ANOVA). Logistic regression was used to test the interactive effects of other variables on the observed association. $\mathrm{P}<0.05$ was considered as being statistically significant.

\section{Results}

Based on the lab data of serum CHE activities retrieved from the clinical lab of our hospital over the past 5 years, the number of cases, mean (standard deviation,SD), median (interquartile ranges) and $P$ values for each of the 48 diseases in comparison to healthy controls were summarized in Table 1. 
Table 1

Serum CHE activities (U/L) in 48 clinically defined diseases in patients and in healthy controls

\begin{tabular}{|c|c|c|c|c|}
\hline CHE & $\begin{array}{l}\text { \# of } \\
\text { cases }\end{array}$ & Mean (SD) & Median (IQR) & $p$ Value \\
\hline Hepatic Encephalopathy & 82 & $3640.8(1221.9)$ & $3633.5(2757.8,4672.0)$ & $<0.001$ \\
\hline Cirrhosis & 8,073 & $4702.4(2321.8)$ & $4032.0(2853.0,6290.0)$ & $<0.001$ \\
\hline Sepsis & 91 & $4830.6(2007.4)$ & $4555.0(3253.0,6366.5)$ & $<0.001$ \\
\hline Pancreatic Cancer & 944 & 5542.7 (2036.8) & $5459.0(4047.0,7144.5)$ & $<0.001$ \\
\hline Liver Cancer & 286 & $6032.7(2163.7)$ & $5939.5(4325.3,7680.5)$ & $<0.001$ \\
\hline Myeloproliferative Disorder & 1,022 & $6113.4(2137.3)$ & $6069.0(4555.5,7743.3)$ & $<0.001$ \\
\hline Chronic Obstructive PD & 1,298 & $6357.7(1966.6)$ & $6321.5(4910.3,7853.3)$ & $<0.001$ \\
\hline Gastric Cancer & 10,414 & $6386.1(1772.5)$ & $6322.5(5098.3,7595.0)$ & $<0.001$ \\
\hline Brain Trauma & 538 & $6492.5(1953.3)$ & $6405.0(5009.8,7872.0)$ & $<0.001$ \\
\hline Uremia & 5,703 & $6388.7(1497.7)$ & $6435.0(5306.5,7437.0)$ & $<0.001$ \\
\hline Esophagus Cancer & 3,060 & $6505.7(1748.8)$ & $6498.5(5181.8,7827.3)$ & $<0.001$ \\
\hline Pancreatitis & 1,376 & $6610.6(2009.6)$ & $6555.5(5207.8,7999.5)$ & $<0.001$ \\
\hline Anemia & 1,792 & $6714.8(2064.1)$ & $6555.0(5201.0,8196.8)$ & $<0.001$ \\
\hline Intracranial Hemorrhage & 3,075 & $6833.2(1911.4)$ & $6754.0(5330.0,8239.0)$ & $<0.001$ \\
\hline Preeclampsia & 728 & $6789.1(1469.1)$ & $6809.5(5766.5,7819.3)$ & $<0.001$ \\
\hline Azotemia & 357 & $6997.0(2067.9)$ & $6921.0(5341.0,8598.0)$ & $<0.001$ \\
\hline Encephalitis & 449 & $7044.4(1949.1)$ & $7023.0(5557.0,8515.0)$ & $<0.001$ \\
\hline Lung Fibrosis & 244 & $7040.6(2013.8)$ & $7049.0(5581.8,8555.3)$ & $<0.001$ \\
\hline Leukemia & 4,228 & $7085.5(2247.5)$ & $7119.5(5387.8,8793.0)$ & $<0.001$ \\
\hline Colon Cancer & 5,279 & 7255.5 (1974.5) & $7267.0(5796.5,8717.0)$ & $<0.001$ \\
\hline $\begin{array}{l}\text { Acute Myocardial } \\
\text { Infarction }\end{array}$ & 1,877 & $7354.7(1806.1)$ & $7378.0(6059.0,8637.0)$ & $<0.001$ \\
\hline Rectum Cancer & 6,663 & $7411.6(1897.1)$ & $7408.0(6022.5,8765.0)$ & $<0.001$ \\
\hline Aplastic Anemia & 816 & $7486.3(2030.8)$ & $7502.5(5932.5,8931.8)$ & $<0.001$ \\
\hline Multiple Myeloma & 1,810 & $7440.5(2291.6)$ & $7710.5(5576.5,9279.8)$ & $<0.001$ \\
\hline
\end{tabular}

Healthy Control is bolded to make easy comparison. SD: standard deviation, IQR: interquartile range. Chronic Obstructive PD: Chronic Obstructive Pulmonary Disease. 


\begin{tabular}{|c|c|c|c|c|}
\hline CHE & $\begin{array}{l}\text { \# of } \\
\text { cases }\end{array}$ & Mean (SD) & Median (IQR) & $p$ Value \\
\hline Bone Fracture & 1,335 & $7693.0(1988.4)$ & $7785.0(6330.5,9198.0)$ & $<0.001$ \\
\hline Acute Cerebral Infarction & 7,108 & $7814.3(1815.8)$ & $7868.0(6594.0,9132.3)$ & $<0.001$ \\
\hline Lymphoma & 3,543 & $7789.0(2409.0)$ & $8037.0(6142.0,9559.5)$ & $<0.001$ \\
\hline Coronary Heart Disease & 16,725 & $8023.3(1955.6)$ & $8190.0(6679.0,9494.0)$ & $<0.001$ \\
\hline Rheumatoid Arthritis & 455 & $8190.0(1750.7)$ & $8278.0(7005.5,9428.3)$ & $<0.001$ \\
\hline Diabetic Nephropathy & 484 & $8131.9(2519.0)$ & $8300.0(6165.3,10103.3)$ & $<0.001$ \\
\hline Asthma & 462 & $8183.7(1731.1)$ & $8326.5(7071.5,9472.8)$ & $<0.001$ \\
\hline Lupus Erythematosus & 1,218 & $8374.4(2004.0)$ & $8463.5(6883.0,9910.5)$ & $<0.001$ \\
\hline Hepatitis & 6,600 & $8204.9(2440.8)$ & $8478.5(6836.0,9834.0)$ & $<0.001$ \\
\hline Cerebral Ischemia & 1,698 & $8434.6(1690.6)$ & $8502.5(7293.8,9646.5)$ & $<0.001$ \\
\hline Lung Cancer & 8,725 & $8477.7(1833.1)$ & $8577.0(7250.0,9798.0)$ & $<0.001$ \\
\hline Nephritis & 1,780 & $8692.2(2181.4)$ & $8689.5(7260.8,10229.3)$ & $<0.001$ \\
\hline Cerebral Arteriosclerosis & 533 & $8551.5(1905.0)$ & $8755.0(7364.0,9888.0)$ & $<0.001$ \\
\hline Gastritis & 2,630 & $8813.8(1676.1)$ & $8819.5(7666.3,10012.3)$ & $<0.001$ \\
\hline Cervical Cancer & 1,800 & $9033.0(1638.2)$ & $9003.5(7869.5,10195.0)$ & $<0.001$ \\
\hline Kidney Cancer & 1,295 & $9002.3(1752.8)$ & $9055.0(7894.5,10116.0)$ & $<0.001$ \\
\hline Type 2 Diabetes Mellitus & 8,803 & $9018.4(1840.8)$ & $9086.0(7805.5,10341.5)$ & $<0.001$ \\
\hline Ovarian Cancer & 1,966 & $8955.8(1989.5)$ & $9133.5(7686.8,10398.8)$ & $<0.001$ \\
\hline Gout & 875 & $9055.4(1931.6)$ & $9228.0(7727.0,10483.0)$ & $<0.001$ \\
\hline Ankylosing Spondylitis & 95 & $9033.3(2122.7)$ & $9417.0(7512.0,10666.5)$ & $<0.001$ \\
\hline \multirow[t]{2}{*}{ Breast Cancer } & \multirow[t]{2}{*}{4584} & 9396.0 & 9435.5(8292.5, & \multirow[t]{2}{*}{$<0.001$} \\
\hline & & $(1566.0)$ & 10505.0) & \\
\hline Endometrial Cancer & 972 & $9346.5(1669.2)$ & $9460.5(8220.8,10599.0)$ & $<0.001$ \\
\hline Psoriasis & 130 & $9281.7(1828.4)$ & $9541.5(7748.5,10630.0)$ & $<0.001$ \\
\hline \multirow[t]{2}{*}{ Nephrotic Syndrome } & \multirow[t]{2}{*}{3284} & 9807.8 & 9639.5(8000.5, & \multirow[t]{2}{*}{$<0.001$} \\
\hline & & $(2455.5)$ & 11440.3) & \\
\hline
\end{tabular}

Healthy Control is bolded to make easy comparison. SD: standard deviation, IQR: interquartile range. Chronic Obstructive PD: Chronic Obstructive Pulmonary Disease. 


\begin{tabular}{|c|c|c|c|c|}
\hline CHE & $\begin{array}{l}\text { \# of } \\
\text { cases }\end{array}$ & Mean (SD) & Median (IQR) & $p$ Value \\
\hline Healthy Control & 3,387 & $\begin{array}{l}10055.3 \\
(1548.4)\end{array}$ & $\begin{array}{l}\text { 10051.0 (8919.0, } \\
11159.5)\end{array}$ & - \\
\hline
\end{tabular}

All 48 different types of diseases had reduced levels of serum CHE activities in comparison to that in healthy controls with statistical significance ( $p<0.001$,Table 1$)$. Among the 48 diseases, patients diagnosed with HE had the lowest median levels of CHE activity, which were followed by cirrhosis, sepsis, pancreatic cancer and liver cancer (Table 1).

To evaluate the diagnostic properties of serum CHE activities as serum biomarkers, the receiving operator curve (ROC) analysis was performed for 48 different types of diseases (Supplemental Fig. 1). Based on the ROC analysis, the area under the curve (AUC), accuracy, sensitivity and specificity for all diseases were listed in Fig. 1. Among the 48 types of diseases studied, 39 of them had the AUCs over 0.60 ranging from 0.60 to 1.00. Remarkably, the serum CHE activity was the best biomarker for HE among all 48 diseases with the AUC of 1.00 , sensitivity $100 \%$, and specificity $99 \%$, which were followed by sepsis (AUC $=0.97$ ), liver cancer (AUC $=0.92)$, myeloproliferative disorder $(A U C=0.92)$, and pancreatitis $(A U C=0.88)$. Interestingly, the serum CHE activities had the lowest AUCs for breast cancer and nephrotic syndrome, being 0.51 and 0.52 , respectively.

We further noticed that all kidney-related diseases including diabetic nephropathy, gout, kidney cancer, nephritis, lupus erythematous, and nephrotic syndrome and all female-related cancers including cervical, ovarian, and breast cancers had relatively low sensitivities (0.14-0.43) and high specificities (0.79-0.95) when $\mathrm{CHE}$ activities were calculated as serum biomarkers (Fig. 1).

To comprehend the observations, we divided the 48 diseases into 6 major categories as cancers, autoimmune diseases, cardio- and cerebrovascular diseases, blood-related diseases (including blood cancers), kidney diseases, and others with different color-code. To visualize the results, we made boxed plots of serum CHE with lower quartile (25\%), median (50\%), and upper quartile (75\%) ranges, and $95 \%$ confident intervals marked for 48 clinically defined human diseases and healthy controls and shown in Fig. 2.

HE had not only the lowest median level (50\%) but also the lowest $2.5 \%, 25 \%, 75 \%$, and $95 \%$ of serum $\mathrm{CHE}$ activities among all diseases. In contrast, nephrotic syndrome was the only disease that had $75 \%$ of serum CHE activities higher than that of control. In addition, diabetic nephropathy, hepatitis, and nephritis were the three diseases that had $97.5 \%$ of serum CHE activities higher than that of control. These observations led to speculate that the extreme serum CHE activities were the characteristics of specific diseases. We then assumed that further studying such a relationship might reveal their relationship with HE and cirrhosis. 
Thus, we analyzed the statistical features of serum CHE activities in six classes of diseases against HE and cirrhosis according to mean values, standard deviation, $2.5,25,50,75$, and 97.5 percentiles. The results of two component analysis of all 48 diseases were shown in Fig. 3.

The apparent clustering of the same category of diseases, such as cancers (at upper side of chart), autoimmune disease (at left side of chart), blood-related diseases (at low right of chart) based on the statistical analysis. Except for HE, diseases directly related to the kidney, such as nephritis, diabetic nephropathy and nephrotic syndrome, were clustered at the low left side of chart. Most impotantly, the two component analysis revealed $\mathrm{HE}$ resembled preeclampsia and uremia whereas cirrhosis resembled multiple myeloma, leukemia, myeloproliferative disorder, and liver cancer as shown in Fig. 3.

\section{Discussion}

All 48 different types of diseases studied in the current study had the lower median serum CHE activities than that of healthy controls (Table 1), implicating that decreased serum $\mathrm{CHE}$ might be a common feature of human diseases. Most of publications reported the dynamic changes of serum CHE activities in one specific disease[32-40], we performed the systematical comparison of serum CHE activities (Table 1) and CHE as biomarkers (Fig. 1) in 48 different types of diseases for the first time. Moreover, serum CHE had almost perfect AUC, accuracy, sensitivity and specificity for HE diagnosis (Fig. 2). Meanwhile, serum CHE activities were also decent serum biomarkers for liver cirrhosis, sepsis and pancreatitis (Fig. 2). Furthermore, the two component analysis results shown in supplemental Fig. 3 and

Fig. 3 revealed the resemblance of $\mathrm{HE}$ to preeclampsia and uremia were more than that to cirrhosis, which provided new insight in understanding HE etiology beyond liver failure. Thus, understanding the molecular mechanism behind the data presented in current study may be helpful in discovering new molecular targets for HE prevention and treatment.

Increased blood ammonia levels have been considered as the main pathophysiological mechanism of $\mathrm{HE}[41,42]$ by causing swelling astrocytes, activation of microglia and neuroinflammation [43-50]. Microglia activation can promote the proliferation and release of pro-inflammatory cytokines[50], and the inflammation state can eventually lead to neuronal death[51]. In addition, astrocytes cultivated with proinflammatory cytokines and interferon gamma (INF-y) becomes edematous, which indicates the potential function of pro-inflammatory cytokines in the process $[52,53]$. Several studies have shown that severe systemic inflammation can induce [54-56] and aggravate HE in cirrhosis[57]. Coltart et al [58] demonstrated that sepsis can induce HE by affecting blood ammonia metabolism and promoting the release of pro-inflammatory mediators. The current study showed that the resenblance of HE to sepsis (Fig. 3), which is consistent with their conclusion.

Inflammation plays a vital role in neurodegenerative diseases[51, 59], such as Alzheimer's disease[60], dementia with Lewy bodies[61], Wilson's disease[62], Parkinson's disease[63], multiple sclerosis[64]. Serum CHE activities is closely related to the inflammation and is involved in many pathophysiological processes of nervous system diseases[65]. Darreh-Shori et al[66] have shown that serum CHE plays an 
important role in regulating intrathecal cytokine and activity of cholinoceptive glial cells in Alzheimer's disease. Macdonald et al [67] showed that CHE has good sensitivity and specificity in the diagnosis of Alzheimer's disease. However, the retrospective CHE activities retrieved in our current study did not provide enough independent test results for other neurodegenerative diseases. Further prospective studies should be useful in addresing these issues.

Chronic hepatitis, especially chronic hepatitis $B$, is the most common cause of cirrhosis and hepatic cancer in China. Hepatic diseases usually progress from hepatitis to cirrhosis and hepatic cancer. Previous studies have shown that CHE levels of hepatitis and cirrhosis are lower than that in healthy controls, which is consistent with the result of the study. Our data showed that serum CHE levels in HE was the lowest not only in liver-related diseases [68]but also in all other diseases included in current study.

\section{Conclusions}

The current study shows that serum CHE was an almost perfect serum biomarker for patients suffering $\mathrm{HE}$ at all stages. The resemblance of HE to preeclampsia and uremia based on CHE activities provided new insight in understanding HE beyond liver failure. The etiology of HE deserves further exploration.

\section{Abbreviations}

CHE:Serum cholinesterase activity; HE:Hepatic encephalopathy; AUC:Area Under the Curve; ROC:Receiving Operator Curve; SD:Standard Deviation; IQR: Interquartile Range; Chronic Obstructive PD: Chronic Obstructive Pulmonary Disease; NF-y:Interferon gamma

\section{Declarations}

\section{Ethics approval and consent to participate}

The Hospital Ethics Review Board of Qingdao University approved current study. All research was performed in accordance with relevant guidelines/regulations and informed consent was obtained from all participants and/or their legal guardians.

\section{Consent for publication}

All authors read and approved the manuscript and the final submission.

Availability of data and materials. All data files are available upon request. Correspondence and requests for the data files should be addressed to H.P and L.Z.

Data analysis. Correspondence and requests for detailed data analysis should be addressed to H.P, Y.G., and L.Z. 


\section{Competing interests}

All authors declare that they have no competing interests.

\section{Funding}

This research was supported by the Natural Science Foundation of China (Grants 81672585 and 11804151), and the Taishan Scholar Fellowship to L.Z.

Authors' contributions Y. R., M.Z., Y.Z., Y.Z., L.Z., Y.G., and H.P had full access to all data in the study and takes responsibility for integrity of the data and accuracy of the data analysis. Concept and design: Y.R., M.Z., L.Z., Y.G., and H.P ; acquisition, analysis or interpretation of data: Y. R., M.Z., Y.Z., Y.Z., L.Z., Y.G., and H.P; statistical analysis: Y.R., M.Z., Y.Z., Y.G., and L.Z.; obtaining funding: L.Z. and Y.G.

Ackowledgements We thank all the stuff and patients in our hospital who made current study possible. We are also grateful to all members of the Systems Biology \& Medicine Center for Complex Diseases who have helped the project in various ways.

\section{References}

1. Ferenci P. Hepatic encephalopathy. Gastroenterol Rep (Oxf). 2017; 5: 138-147.

2. Wijdicks E F. Hepatic Encephalopathy. N Engl J Med. 2016; 375: 1660-1670.

3. Dadsetan S,Balzano T,Forteza J,Agusti A,Cabrera-Pastor A,Taoro-Gonzalez L,Hernandez-Rabaza V,Gomez-Gimenez B,EIMlili N,Llansola M, et al. Infliximab reduces peripheral inflammation, neuroinflammation, and extracellular GABA in the cerebellum and improves learning and motor coordination in rats with hepatic encephalopathy. Journal of neuroinflammation. 2016; 131: 245.

4. Agusti A,Hernández-Rabaza V,Balzano T,Taoro-Gonzalez L,Ibañez-Grau A,Cabrera-Pastor A,Fustero S,Llansola M,Montoliu C,Felipo V. Sildenafil reduces neuroinflammation in cerebellum, restores GABAergic tone, and improves motor in-coordination in rats with hepatic encephalopathy. CNS neuroscience \& therapeutics. 2017; 23: 386-394.

5. Shawcross D,Wright G,Olde Damink S,Jalan R. Role of ammonia and inflammation in minimal hepatic encephalopathy. Metab Brain Dis 2007; 221: 125-38.

6. Kaji K,Takaya H,Saikawa S,Furukawa M,Sato S,Kawaratani H,Kitade M,Moriya K,Namisaki T,Akahane $\mathrm{T}$, et al. Rifaximin ameliorates hepatic encephalopathy and endotoxemia without affecting the gut microbiome diversity. World journal of gastroenterology. 2017; 2347: 8355-8366.

7. Zamanian G,Partoazar A,Tavangar S,Rashidian A,Mirzaei P,Niaz Q,Sharifi K,Dehpour A,Jazaeri F. Effect of phosphatidylserine on cirrhosis-induced hepatic encephalopathy: Response to acute endotoxemia in cirrhotic rats. Life sciences. 2020; 253: 117606.

8. Vilstrup H,Amodio P,Bajaj J,Cordoba J,Ferenci P,Mullen K,Weissenborn K,Wong P. Hepatic encephalopathy in chronic liver disease: 2014 Practice Guideline by the American Association for the 
Study of Liver Diseases and the European Association for the Study of the Liver. Hepatology (Baltimore, Md.). 2014; 60: 715-35.

9. Fichet J,Mercier E,Genée 0,Garot D,Legras A,Dequin P,Perrotin D. Prognosis and 1-year mortality of intensive care unit patients with severe hepatic encephalopathy. J Crit Care 2009; 243: 364-370.

10. Vilstrup H,Amodio P,Bajaj J,Cordoba J,Ferenci P,Mullen K,Weissenborn K,Wong P. Hepatic encephalopathy in chronic liver disease: 2014 Practice Guideline by the American Association for the Study of Liver Diseases and the European Association for the Study of the Liver. Hepatology 2014; 602: 715-735.

11. Zacharias H,Zacharias A,Gluud L,Morgan M. Pharmacotherapies that specifically target ammonia for the prevention and treatment of hepatic encephalopathy in adults with cirrhosis. The Cochrane database of systematic reviews. 2019; 6: CD012334.

12. Sergeeva O. GABAergic transmission in hepatic encephalopathy. Archives of biochemistry and biophysics. 2013; 5362: 122.

13. Kobtan A,El-Kalla F,Soliman H,Zakaria S,Goda M. Higher Grades and Repeated Recurrence of Hepatic Encephalopathy May Be Related to High Serum Manganese Levels. Biological trace element research. 2016; 1692: 153.

14. Bemeur C,Desjardins P,Butterworth R. Evidence for oxidative/nitrosative stress in the pathogenesis of hepatic encephalopathy. Metabolic brain disease. 2010; 25: 3-9.

15. Tang L,Zhang M,Li X,Zhang L. Glucuronidated bilirubin: Significantly increased in hepatic encephalopathy. Prog Mol Biol Transl Sci. 2019; 162: 363-376.

16. Ninan J,Feldman L. Ammonia Levels and Hepatic Encephalopathy in Patients with Known Chronic Liver Disease. J Hosp Med 2017; 128: 659-661.

17. Ong J,Aggarwal A,Krieger D,Easley K,Karafa M,Van Lente F,Arroliga A,Mullen K. Correlation between ammonia levels and the severity of hepatic encephalopathy. Am J Med. 2003; 1143: 188-93.

18. Bosoi C,Rose C. Oxidative stress: a systemic factor implicated in the pathogenesis of hepatic encephalopathy. Metab Brain Dis. 2013; 282: 175.

19. Lockridge O,Bartels C,Vaughan T,Wong C,Norton S,Johnson L. Complete amino acid sequence of human serum cholinesterase. The Journal of biological chemistry. 1987; 2622: 549-557.

20. Jensen F,Schwartz M,Viby-Mogensen J. Identification of human plasma cholinesterase variants using molecular biological techniques. Acta anaesthesiologica Scandinavica. 1995; 39: 142-9.

21. Li H,Schopfer L,Masson P,Lockridge O. Lamellipodin proline rich peptides associated with native plasma butyrylcholinesterase tetramers. Biochem. J. 2008; 4112: 425-432.

22. De Vriese C,Gregoire F,Lema-Kisoka R,Waelbroeck M,Robberecht P,Delporte C. Ghrelin degradation by serum and tissue homogenates: identification of the cleavage sites. Endocrinology. 2004; 145: $4997-$ 5005.

23. Gorelick D. Enhancing cocaine metabolism with butyrylcholinesterase as a treatment strategy. Drug Alcohol Depend. 1997; 483: 159-165. 
24. Lockridge 0 . Genetic variants of human serum cholinesterase influence metabolism of the muscle relaxant succinylcholine. Pharmacology \& therapeutics. 1990; 471: 35-60.

25. Lockridge 0,Masson P. Pesticides and susceptible populations: people with butyrylcholinesterase genetic variants may be at risk. Neurotoxicology. 2000; 21: 113-26.

26. Ashani Y,Shapira S,Levy D,Wolfe A,Doctor B,Raveh L. Butyry/cholinesterase and acetylcholinesterase prophylaxis against soman poisoning in mice. Biochemical pharmacology. 1991; 411: 37-41.

27. Cerejeira J,Nogueira V,Luís P,Vaz-Serra A,Mukaetova-Ladinska E. The cholinergic system and inflammation: common pathways in delirium pathophysiology. J Am Geriatr Soc. 2012; 604: 669675.

28. Shenhar-Tsarfaty S,Berliner S,Bornstein N,Soreq H. Cholinesterases as biomarkers for parasympathetic dysfunction and inflammation-related disease. J Mol Neurosci. 2014; 533: 298-305.

29. Miao Y,He N,Zhu J. History and new developments of assays for cholinesterase activity and inhibition. Chem. Rev. 2010; 1109: 5216-5234.

30. ELLMAN G,COURTNEY K,ANDRES V,FEATHER-STONE R. A new and rapid colorimetric determination of acetylcholinesterase activity. Biochem. Pharmacol. 1961; 7: 88-95.

31. Arduini F,Errico I,Amine A,Micheli L,Palleschi G,Moscone D. Enzymatic spectrophotometric method for aflatoxin B detection based on acety/cholinesterase inhibition. Anal. Chem. 2007; 799: 3409-3415.

32. Zivkovic A,Schmidt K,Sigl A,Decker S,Brenner T,Hofer S. Reduced serum butyrylcholinesterase activity indicates severe systemic inflammation in critically ill patients. Mediators Inflamm. 2015.

33. Shenhar-Tsarfaty S,Bruck T,Bennett E,Bravman T,Aassayag E,Waiskopf N,Rogowski O,Bornstein $\mathrm{N}$,Berliner S,Soreq $\mathrm{H}$. Butyry/cholinesterase interactions with amylin may protect pancreatic cells in metabolic syndrome. J. Cell. Mol. Med. 2011; 158: 1747-1756.

34. Goliasch G,Haschemi A,Marculescu R,Endler G,Maurer G,Wagner O,Huber K,Mannhalter C,Niessner A. Butyrylcholinesterase activity predicts long-term survival in patients with coronary artery disease. Clin. Chem. 2012; 586: 1055-8.

35. Sicinska P,Bukowska B,Pajak A,Koceva-Chyla A,Pietras T,Nizinkowski P,Gorski P,Koter-Michalak M. Decreased activity of butyrylcholinesterase in blood plasma of patients with chronic obstructive pulmonary disease. Archives of medical science : AMS. 2017; 133: 645-651.

36. Montenegro M,Ruiz-Espejo F,Campoy F,Muñoz-Delgado E,de la Cadena M,Cabezas-Herrera J,Vidal C. Acetyl- and butyrylcholinesterase activities decrease in human colon adenocarcinoma. Journal of molecular neuroscience: MN. 2006; 30.

37. Klocker E,Barth D,Riedl J,Prinz F,Szkandera J,Schlick K,Kornprat P,Lackner K,Lindenmann J,Stöger H, et al. Decreased Activity of Circulating Butyry/cholinesterase in Blood Is an Independent Prognostic Marker in Pancreatic Cancer Patients. Cancers (Basel) 2020; 125.

38. Koie T,Ohyama C,Yamamoto H,Hatakeyama S,Imai A,Yoneyama T,Hashimoto Y,Kitayam M,Hirota K. Significance of preoperative butyry/cholinesterase as an independent predictor of survival in patients with muscle-invasive bladder cancer treated with radical cystectomy. Urologic oncology. 2014; 326. 
39. Battisti V,Bagatini M,Maders L,Chiesa J,Santos K,Gonçalves J,Abdalla F,Battisti I,Schetinger $\mathrm{M}$, Morsch V. Cholinesterase activities and biochemical determinations in patients with prostate cancer: influence of Gleason score, treatment and bone metastasis. Biomedicine \& pharmacotherapy = Biomedecine \& pharmacotherapie. 2012; 66: 249-55.

40. Coulter D,Boettner A,Kortylewicz Z,Enke S,Luther J,Verma V,Baranowska-Kortylewicz J. Butyrylcholinesterase as a Blood Biomarker in Neuroblastoma. Journal of pediatric hematology/oncology. 2017; 394: 272-281.

41. Donovan J,Schafer D,Shaw B,Sorrell M. Cerebral oedema and increased intracranial pressure in chronic liver disease. Lancet (London, England). 1998; 351: 719-21.

42. Fiati Kenston S,Song X,Li Z,Zhao J. Mechanistic insight, diagnosis, and treatment of ammoniainduced hepatic encephalopathy. J Gastroenterol Hepatol. 2019; 341: 31-39.

43. McMillin M,Grant S,Frampton G,Andry S,Brown A,DeMorrow S. Fractalkine suppression during hepatic encephalopathy promotes neuroinflammation in mice. J Neuroinflammation. 2016; 131: 198.

44. Rodrigo R,Cauli O,Gomez-Pinedo U,Agusti A,Hernandez-Rabaza V,Garcia-Verdugo J,Felipo V. Hyperammonemia induces neuroinflammation that contributes to cognitive impairment in rats with hepatic encephalopathy. Gastroenterology. 2010; 1392: 675-684.

45. El Khiat A,Tamegart L,Draoui A,El Fari R,Sellami S,Rais H,El Hiba O,Gamrani H. Kinetic deterioration of short memory in rat with acute hepatic encephalopathy: Involvement of astroglial and neuronal dysfunctions. Behav Brain Res. 2019; 367: 201-209.

46. Ding S,Wang X,Zhuge W,Yang J,Zhuge Q. Dopamine induces glutamate accumulation in astrocytes to disrupt neuronal function leading to pathogenesis of minimal hepatic encephalopathy. Neuroscience. 2017; 365: 94-113.

47. Blei A,Olafsson S,Therrien G,Butterworth R. Ammonia-induced brain edema and intracranial hypertension in rats after portacaval anastomosis. Hepatology. 1994; 619: 1437-44.

48. Norenberg M,Martinez-Hernandez A. Fine structural localization of glutamine synthetase in astrocytes of rat brain. Brain Res. 1979; 1612: 303-10.

49. Montes-Cortes D,Olivares-Corichi I,Rosas-Barrientos J,Manuel-Apolinar L,Martìnez-Godinez M,Hernández-López J,Cruz-Dominguez M. Characterization of Oxidative Stress and Ammonia According to the Different Grades of Hepatic Encephalopathy. Digestive diseases (Basel, Switzerland). 2020; 383: 240-250.

50. Bezzi P,Domercq M,Vesce S,Volterra A. Neuron-astrocyte cross-talk during synaptic transmission: physiological and neuropathological implications. Prog Brain Res 2001; 132: 255-65.

51. McCoy M,Tansey M. TNF signaling inhibition in the CNS: implications for normal brain function and neurodegenerative disease. J Neuroinflammation. 2008; 5: 45.

52. Wright G,Jalan R. Ammonia and inflammation in the pathogenesis of hepatic encephalopathy: Pandora's box? Hepatology. 2007; 462: 291-4.

53. Butterworth R. The liver-brain axis in liver failure: neuroinflammation and encephalopathy. Nat Rev Gastroenterol Hepatol. 2013; 10: 522-8. 
54. Shawcross D,Shabbir S,Taylor N,Hughes R. Ammonia and the neutrophil in the pathogenesis of hepatic encephalopathy in cirrhosis. Hepatology. 2010; 513: 1062-9.

55. Shawcross D,Wright G,Olde Damink S,Jalan R. Role of ammonia and inflammation in minimal hepatic encephalopathy. Metab Brain Dis. 2007; 221: 125-38.

56. Aldridge D,Tranah E,Shawcross D. Pathogenesis of hepatic encephalopathy: role of ammonia and systemic inflammation. Journal of clinical and experimental hepatology. 2015; 5: S7-S20.

57. Manzhalii E,Virchenko O,Falalyeyeva T,Moiseienko V,Nykula T,Kondratiuk V,Savchuk O,Beregova T,Stremmel W. Hepatic Encephalopathy Aggravated by Systemic Inflammation. Dig Dis. 2019; 376: 509-517.

58. Coltart I,Tranah T,Shawcross D. Inflammation and hepatic encephalopathy. Archives of biochemistry and biophysics. 2013; 5362: 189-96.

59. Ellwardt E,Zipp F. Molecular mechanisms linking neuroinflammation and neurodegeneration in MS. Experimental neurology. 2014: 8-17.

60. Agarwal A,Mais D. Sensitivity and Specificity of Alzheimer Type II Astrocytes in Hepatic Encephalopathy. Arch Pathol Lab Med. 2019; 14310: 1256-1258.

61. Surendranathan A,Su L,Mak E,Passamonti L,Hong Y,Arnold R,Vázquez Rodríguez P,Bevan-Jones W,Brain S,Fryer T, et al. Early microglial activation and peripheral inflammation in dementia with Lewy bodies. Brain : a journal of neurology. 2018; 14112: 3415-3427.

62. Medici V,Shibata N,Kharbanda K,LaSalle J,Woods R,Liu S,Engelberg J,Devaraj S,Török N,Jiang J, et al. Wilson's disease: changes in methionine metabolism and inflammation affect global DNA methylation in early liver disease. Hepatology (Baltimore, Md.). 2013; 572: 555-65.

63. King E,O'Brien J,Donaghy P,Williams-Gray C,Lawson R,Morris C,Barnett N,Olsen K,Martin-Ruiz C,Burn $\mathrm{D}$, et al. Inflammation in mild cognitive impairment due to Parkinson's disease, Lewy body disease, and Alzheimer's disease. International journal of geriatric psychiatry. 2019; 34: 1244-1250.

64. Baird J,Bourdette D,Meshul C,Quinn J. The key role of T cells in Parkinson's disease pathogenesis and therapy. Parkinsonism \& related disorders. 2019; 60: 25-31.

65. Shenhar-Tsarfaty S,Berliner S,Bornstein N,Soreq H. Cholinesterases as biomarkers for parasympathetic dysfunction and inflammation-related disease. Journal of molecular neuroscience : MN. 2014; 533: 298-305.

66. Darreh-Shori T,Vijayaraghavan S,Aeinehband S,Piehl F,Lindblom R,Nilsson B,Ekdahl K,Långström B,Almkvist O,Nordberg A. Functional variability in butyrylcholinesterase activity regulates intrathecal cytokine and astroglial biomarker profiles in patients with Alzheimer's disease. Neurobiology of aging. 2013; 3411: 2465-81.

67. Macdonald I,Maxwell S,Reid G,Cash M,DeBay D,Darvesh S. Quantification of Butyrylcholinesterase Activity as a Sensitive and Specific Biomarker of Alzheimer's Disease. Journal of Alzheimer's disease : JAD. 2017; 58: 491-505.

68. Liu T,Yuan C. Severe depression of serum cholinesterase activity associated with two cases of hepatic encephalopathy. Clin. Chem. 1985; 319: 1570. 
Figures

\begin{tabular}{|c|c|c|c|c|}
\hline 1.00 & 0.99 & 1.00 & 0.99 & Hepatic Encephalopathy \\
\hline 0.97 & 0.93 & 0.91 & 0.96 & Sepsis \\
\hline 0.92 & 0.84 & 0.83 & 0.85 & - Liver Cancer \\
\hline 0.92 & 0.84 & 0.77 & 0.91 & Myeloproliferative Disorder \\
\hline 0.88 & 0.81 & 0.76 & 0.87 & Pancreatitis \\
\hline 0.88 & 0.81 & 0.79 & 0.83 & Brain Trauma \\
\hline 0.87 & 0.82 & 0.80 & 0.83 & Pancreatic Cancer \\
\hline 0.86 & 0.78 & 0.70 & 0.85 & Azotemia \\
\hline 0.86 & 0.79 & 0.66 & 0.91 & Lung Fibrosis \\
\hline 0.82 & 0.76 & 0.70 & 0.82 & Encephalitis \\
\hline 0.79 & 0.74 & 0.65 & 0.81 & Anemia \\
\hline 0.77 & 0.70 & 0.76 & 0.66 & Intracranial Hemorrhage \\
\hline 0.76 & 0.68 & 0.86 & 0.57 & Uremia \\
\hline 0.75 & 0.70 & 0.62 & 0.77 & Bone Fracture \\
\hline 0.75 & 0.69 & 0.69 & 0.69 & Asthma \\
\hline 0.75 & 0.70 & 0.58 & 0.79 & Aplastic Anemia \\
\hline 0.74 & 0.67 & 0.53 & 0.81 & Rheumatoid Arthritis \\
\hline 0.73 & 0.66 & 0.76 & 0.60 & Acute Myocardial Infarction \\
\hline 0.72 & 0.68 & 0.66 & 0.69 & Leukemia \\
\hline 0.71 & 0.67 & 0.62 & 0.71 & Multiple Myeloma \\
\hline 0.71 & 0.69 & 0.43 & 0.95 & Diabetic Nephropathy \\
\hline 0.70 & 0.59 & 0.86 & 0.47 & Esophagus Cancer \\
\hline 0.70 & 0.62 & 0.83 & 0.52 & Chronic Obstructive PD \\
\hline 0.69 & 0.61 & 0.79 & 0.54 & Cirrhosis \\
\hline 0.67 & 0.62 & 0.69 & 0.58 & Preeclampsia \\
\hline 0.67 & 0.63 & 0.59 & 0.66 & Cerebral Arteriosclerosis \\
\hline 0.66 & 0.63 & 0.35 & 0.89 & Gout \\
\hline 0.66 & 0.65 & 0.51 & 0.74 & Lymphoma \\
\hline 0.66 & 0.63 & 0.69 & 0.56 & Ankylosing Spondylitis \\
\hline 0.66 & 0.64 & 0.49 & 0.74 & Cerebral Ischemia \\
\hline 0.65 & 0.61 & 0.43 & 0.79 & Kidney Cancer \\
\hline 0.65 & 0.57 & 0.70 & 0.52 & Colon Cancer \\
\hline 0.65 & 0.61 & 0.59 & 0.62 & Gastritis \\
\hline 0.63 & 0.63 & 0.39 & 0.83 & Nephritis \\
\hline 0.63 & 0.55 & 0.71 & 0.49 & Rectum Cancer \\
\hline 0.62 & 0.51 & 0.75 & 0.44 & Gastric Cancer \\
\hline 0.62 & 0.58 & 0.61 & 0.56 & Hepatitis \\
\hline 0.61 & 0.59 & 0.68 & 0.51 & Psoriasis \\
\hline 0.60 & 0.62 & 0.38 & 0.79 & Lupus Erythematosus \\
\hline 0.59 & 0.47 & 0.76 & 0.38 & Acute Cerebral Infarction \\
\hline 0.56 & 0.57 & 0.49 & 0.60 & Lung Cancer \\
\hline 0.55 & 0.62 & 0.19 & 0.89 & Cervical Cancer \\
\hline 0.55 & 0.54 & 0.53 & 0.55 & Type 2 Diabetes Mellitus \\
\hline 0.55 & 0.43 & 0.70 & 0.38 & Coronary Heart Disease \\
\hline 0.54 & 0.59 & 0.14 & 0.93 & Endometrial Cancer \\
\hline 0.53 & 0.63 & 0.22 & 0.86 & Ovarian Cancer \\
\hline 0.52 & 0.61 & 0.26 & 0.85 & Nephrotic Syndrome \\
\hline 0.51 & 0.68 & 0.21 & 0.81 & Breast Cancer \\
\hline
\end{tabular}

\section{Figure 1}

The AUC, accuracy, sensitivity and specificity of serum CHE activities for 48 types of human diseases. AUC: Area under the curve. Chronic Obstructive PD: Chronic Obstructive Pulmonary Disease. 


\begin{tabular}{|c|c|c|c|c|}
\hline 1.00 & 0.99 & 1.00 & 0.99 & Hepatic Encephalopathy \\
\hline 0.97 & 0.93 & 0.91 & 0.96 & Sepsis \\
\hline 0.92 & 0.84 & 0.83 & 0.85 & Liver Cancer \\
\hline 0.92 & 0.84 & 0.77 & 0.91 & Myeloproliferative Disorder \\
\hline 0.88 & 0.81 & 0.76 & 0.87 & Pancreatitis \\
\hline 0.88 & 0.81 & 0.79 & 0.83 & Brain Trauma \\
\hline 0.87 & 0.82 & 0.80 & 0.83 & Pancreatic Cancer \\
\hline 0.86 & 0.78 & 0.70 & 0.85 & Azotemia \\
\hline 0.86 & 0.79 & 0.66 & 0.91 & Lung Fibrosis \\
\hline 0.82 & 0.76 & 0.70 & 0.82 & Encephalitis \\
\hline 0.79 & 0.74 & 0.65 & 0.81 & Anemia \\
\hline 0.77 & 0.70 & 0.76 & 0.66 & Intracranial Hemorrhage \\
\hline 0.76 & 0.68 & 0.86 & 0.57 & Uremia \\
\hline 0.75 & 0.70 & 0.62 & 0.77 & Bone Fracture \\
\hline 0.75 & 0.69 & 0.69 & 0.69 & Asthma \\
\hline 0.75 & 0.70 & 0.58 & 0.79 & Aplastic Anemia \\
\hline 0.74 & 0.67 & 0.53 & 0.81 & Rheumatoid Arthritis \\
\hline 0.73 & 0.66 & 0.76 & 0.60 & Acute Myocardial Infarction \\
\hline 0.72 & 0.68 & 0.66 & 0.69 & Leukemia \\
\hline 0.71 & 0.67 & 0.62 & 0.71 & Multiple Myeloma \\
\hline 0.71 & 0.69 & 0.43 & 0.95 & Diabetic Nephropathy \\
\hline 0.70 & 0.59 & 0.86 & 0.47 & Esophagus Cancer \\
\hline 0.70 & 0.62 & 0.83 & 0.52 & Chronic Obstructive PD \\
\hline 0.69 & 0.61 & 0.79 & 0.54 & Cirrhosis \\
\hline 0.67 & 0.62 & 0.69 & 0.58 & Preeclampsia \\
\hline 0.67 & 0.63 & 0.59 & 0.66 & Cerebral Arteriosclerosis \\
\hline 0.66 & 0.63 & 0.35 & 0.89 & Gout \\
\hline 0.66 & 0.65 & 0.51 & 0.74 & Lymphoma \\
\hline 0.66 & 0.63 & 0.69 & 0.56 & Ankylosing Spondylitis \\
\hline 0.66 & 0.64 & 0.49 & 0.74 & Cerebral Ischemia \\
\hline 0.65 & 0.61 & 0.43 & 0.79 & Kidney Cancer \\
\hline 0.65 & 0.57 & 0.70 & 0.52 & Colon Cancer \\
\hline 0.65 & 0.61 & 0.59 & 0.62 & Gastritis \\
\hline 0.63 & 0.63 & 0.39 & 0.83 & Nephritis \\
\hline 0.63 & 0.55 & 0.71 & 0.49 & Rectum Cancer \\
\hline 0.62 & 0.51 & 0.75 & 0.44 & - Gastric Cancer \\
\hline 0.62 & 0.58 & 0.61 & 0.56 & Hepatitis \\
\hline 0.61 & 0.59 & 0.68 & 0.51 & Psoriasis \\
\hline 0.60 & 0.62 & 0.38 & 0.79 & Lupus Erythematosus \\
\hline 0.59 & 0.47 & 0.76 & 0.38 & Acute Cerebral Infarction \\
\hline 0.56 & 0.57 & 0.49 & 0.60 & Lung Cancer \\
\hline 0.55 & 0.62 & 0.19 & 0.89 & Cervical Cancer \\
\hline 0.55 & 0.54 & 0.53 & 0.55 & Type 2 Diabetes Mellitus \\
\hline 0.55 & 0.43 & 0.70 & 0.38 & Coronary Heart Disease \\
\hline 0.54 & 0.59 & 0.14 & 0.93 & Endometrial Cancer \\
\hline 0.53 & 0.63 & 0.22 & 0.86 & Ovarian Cancer \\
\hline 0.52 & 0.61 & 0.26 & 0.85 & Nephrotic Syndrome \\
\hline 0.51 & 0.68 & 0.21 & 0.81 & Breast Cancer \\
\hline
\end{tabular}

\section{Figure 1}

The AUC, accuracy, sensitivity and specificity of serum CHE activities for 48 types of human diseases. AUC: Area under the curve. Chronic Obstructive PD: Chronic Obstructive Pulmonary Disease. 


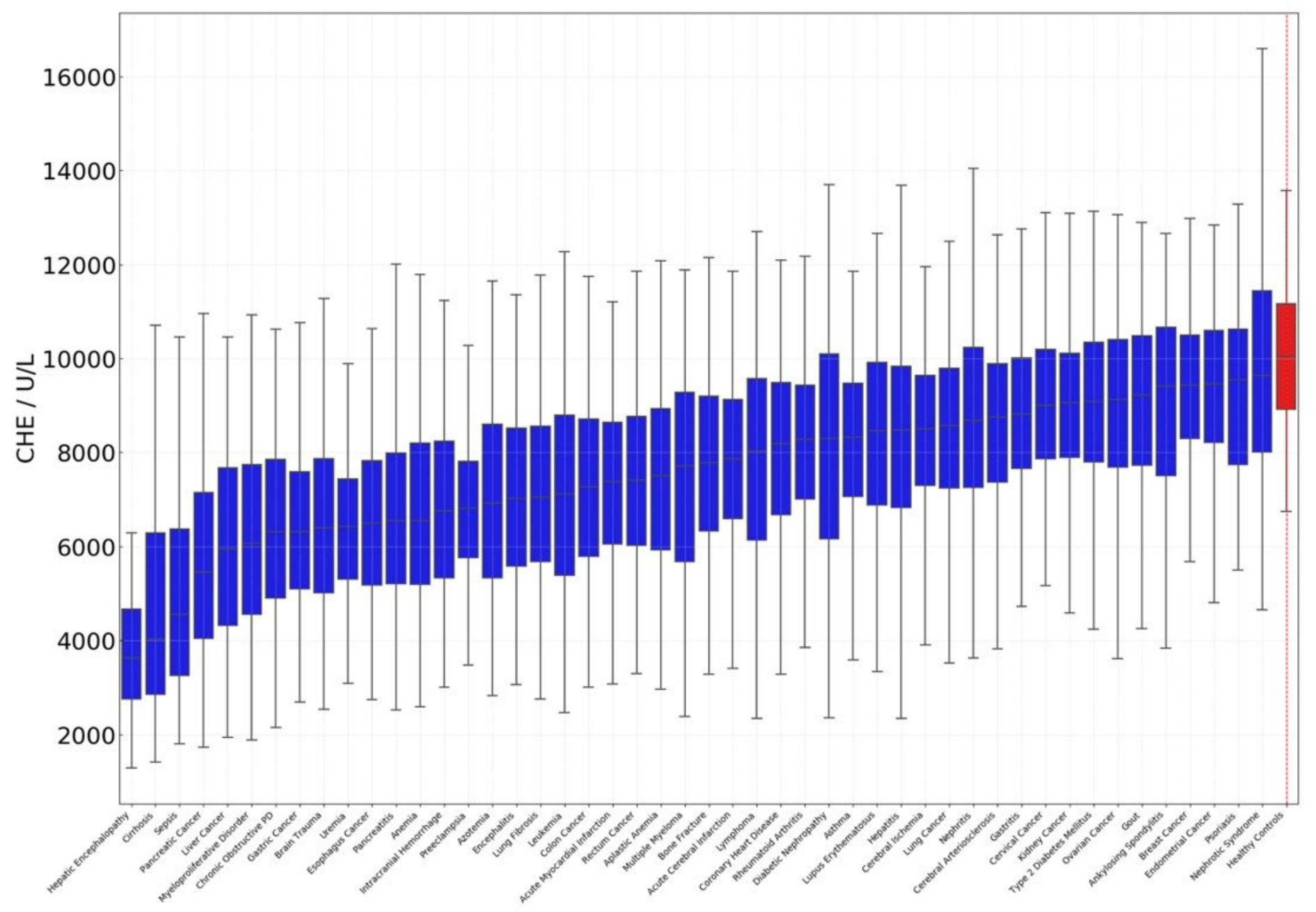

Figure 2

Serum CHE activities in 48 different types of diseases and healthy controls. The data of serum CHE was arranged in an ascending order according to the median values. Chronic Obstructive PD: Chronic Obstructive Pulmonary Disease. 


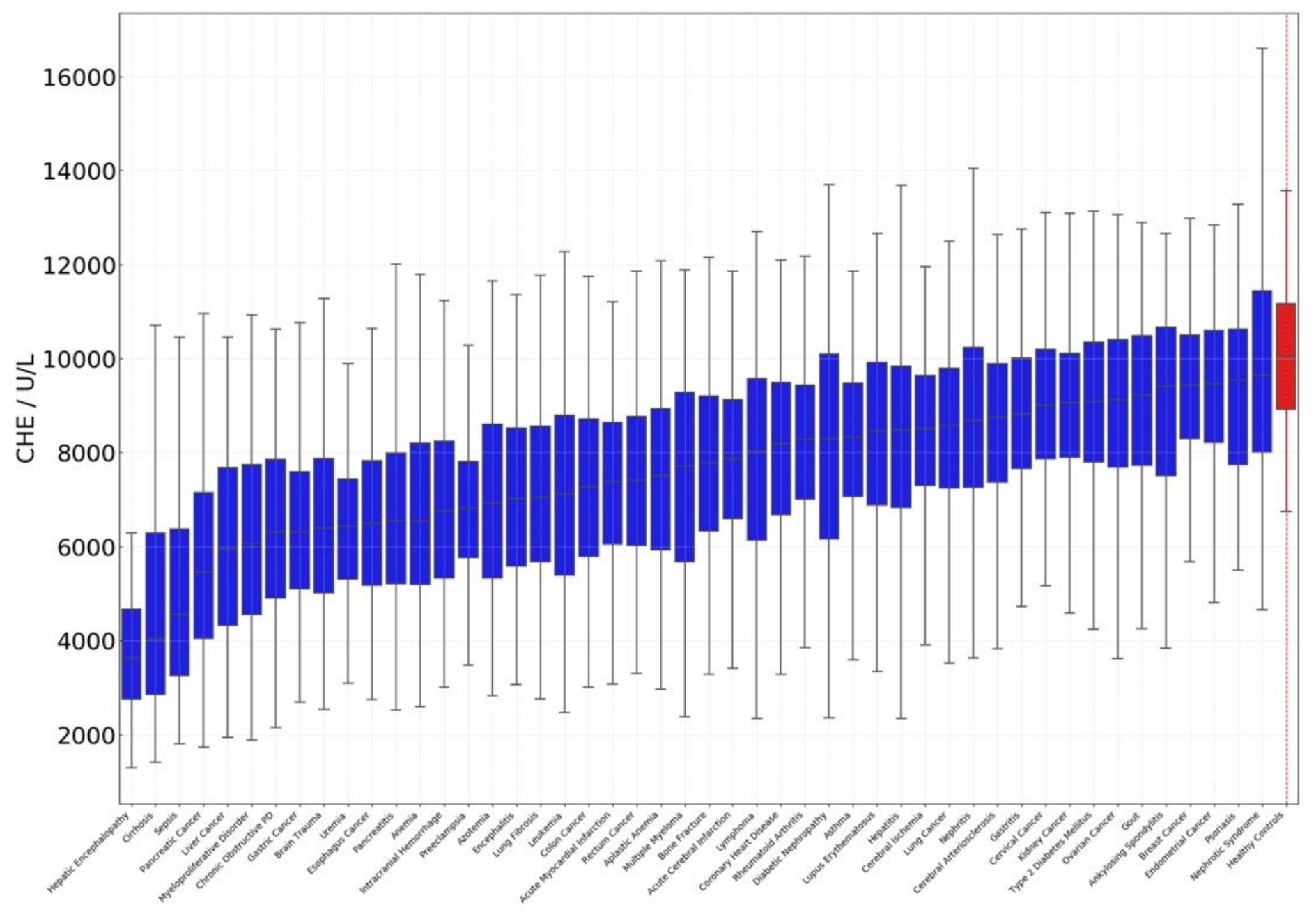

Figure 2

Serum CHE activities in 48 different types of diseases and healthy controls. The data of serum CHE was arranged in an ascending order according to the median values. Chronic Obstructive PD: Chronic Obstructive Pulmonary Disease. 


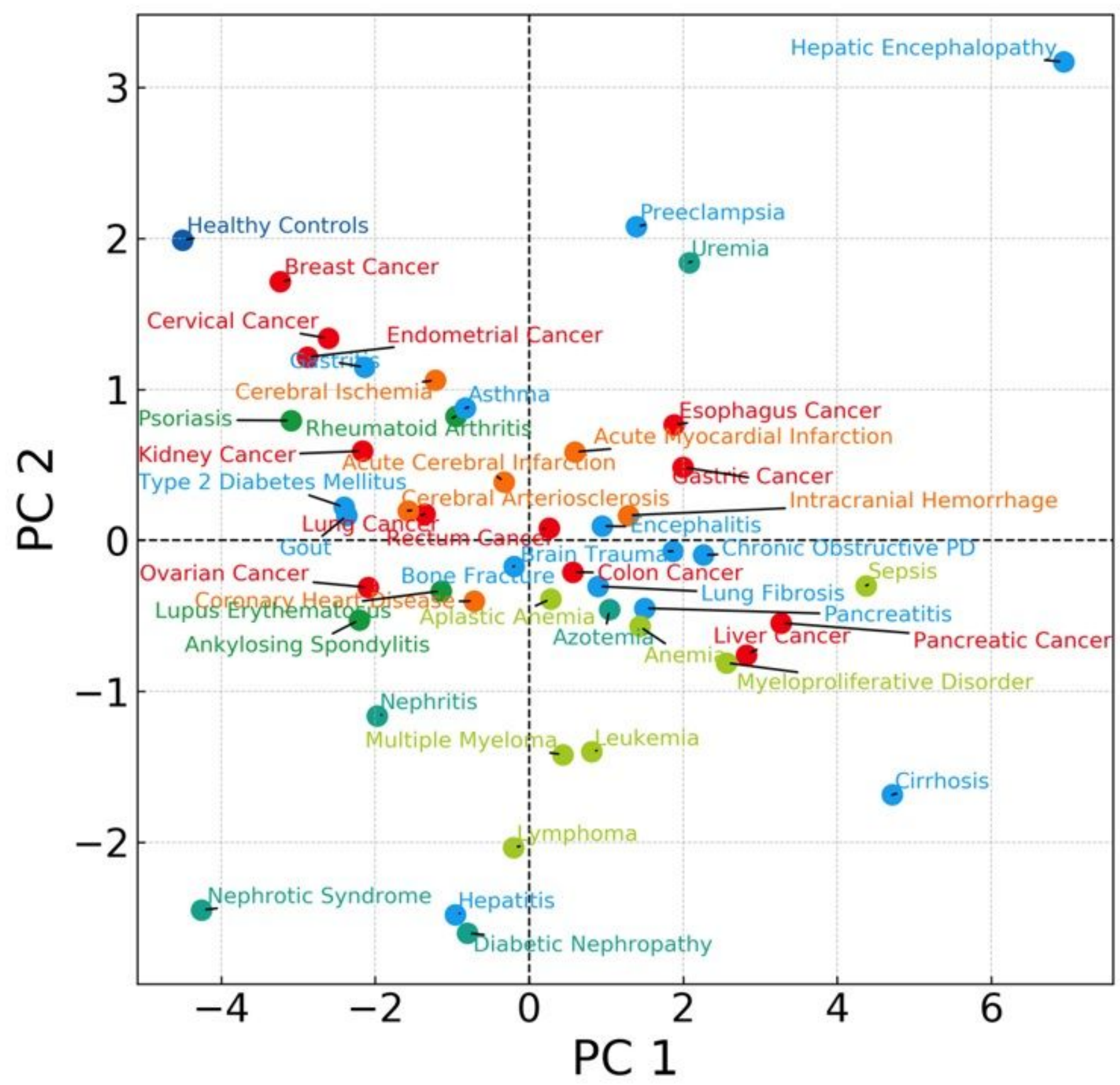

\section{Figure 3}

The serum CHE activities had common features in the same class of diseases. The 48 diseases were grouped into 6 major categories including cancers, autoimmune diseases, cardiovascular and cerebrovascular diseases, blood-related diseases, kidney diseases, and others, being marked with red, green, orange, yellow green, teal, and blue, respectively. The statistical features of serum CHE activities of 48 diseases including the mean values, standard deviation, min/max values, 25, 50, and 75 percentiles were quantified. The obtained statistical features of all diseases were further decomposed into two components and presented. 


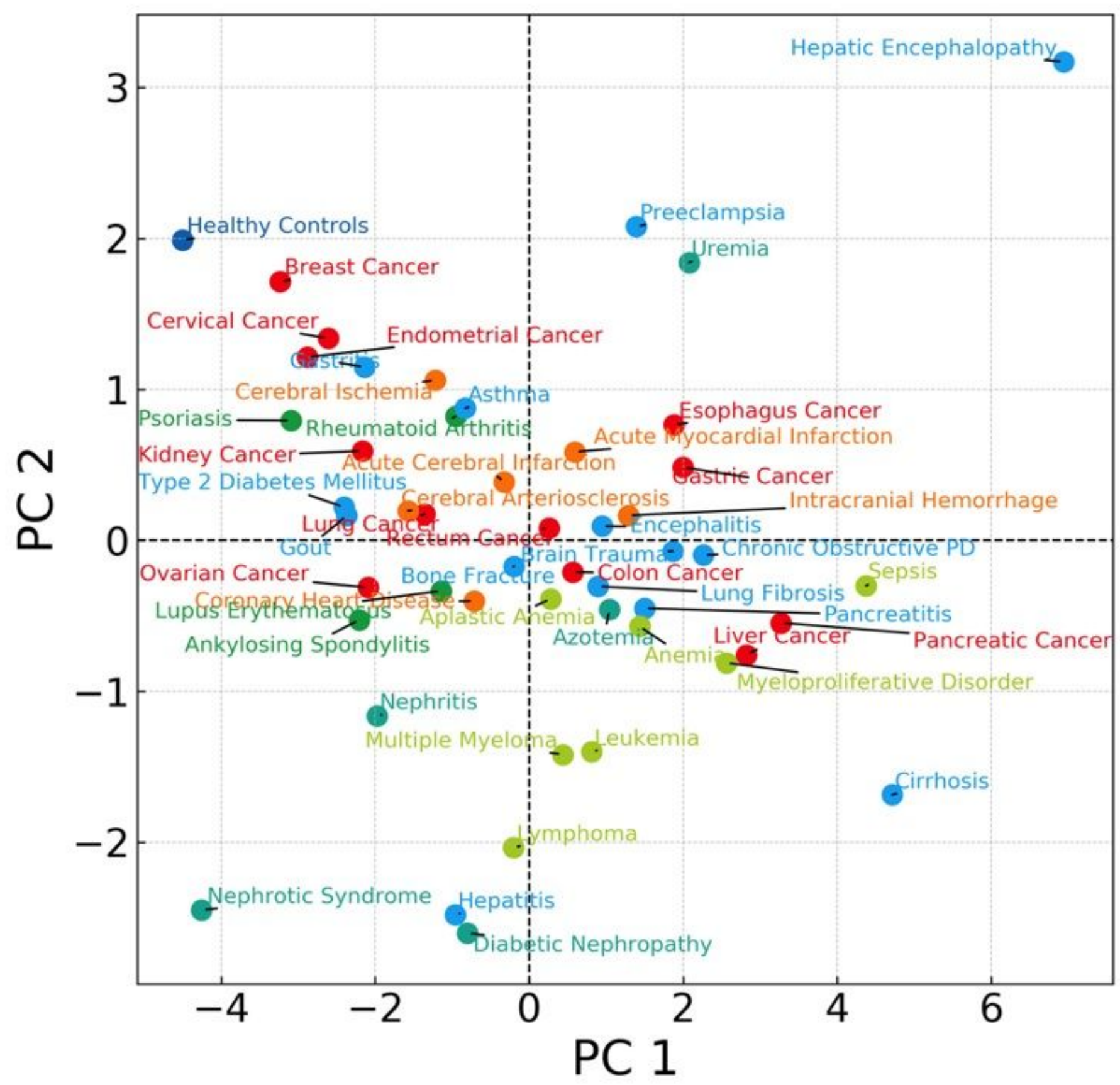

\section{Figure 3}

The serum CHE activities had common features in the same class of diseases. The 48 diseases were grouped into 6 major categories including cancers, autoimmune diseases, cardiovascular and cerebrovascular diseases, blood-related diseases, kidney diseases, and others, being marked with red, green, orange, yellow green, teal, and blue, respectively. The statistical features of serum CHE activities of 48 diseases including the mean values, standard deviation, min/max values, 25, 50, and 75 percentiles were quantified. The obtained statistical features of all diseases were further decomposed into two components and presented. 


\section{Supplementary Files}

This is a list of supplementary files associated with this preprint. Click to download.

- Onlinefloatimage4.Png

- Onlinefloatimage4.Png 